\title{
A nudge and a wink
}

Shaun Sellars continues this exciting and essential series on ethical dilemmas in dentistry which appears in every second issue of the BDJ.

Nudge theory suggests that it's possible to influence people's behaviour by the use of indirect suggestions and positive reinforcement. These 'nudges' could range from simple actions such as subtly changing the phrasing of our advice to making changes to the surroundings and are usually inexpensive and easy to implement. The text message or phone call we make to a patient to remind them of their upcoming appointment... that's a nudge.

If we can build up a set of high powered nudges, we may be able to effect positive behavioural change in patients in a way that's readily acceptable and effective.

Certain types of nudges have shown to be more effective than others. For example, the phrase 'people who have healthy gums tend to have healthy hearts' is powerful because it simplifies the link between heart disease and periodontal disease. It also plays on the idea of the societal norm, and people are more inclined to do as others do. Add some form of picture to this, and you're likely to make the nudge more powerful still.

Nudges that incorporate ideas of societal norms are some of the most powerful influencers of behaviour. For example, if a patient comes in wanting straight white teeth and we tell them that most people in a similar situation choose to have veneers rather than orthodontics, they're more likely to go with the veneer option, simply because it's what most do. We are all birds of a feather.

Of course, there are issues with this form of soft paternalism. One could claim that manipulation of people's behaviour in such covert ways reduces personal autonomy and undermines agency. Defenders of nudge theory would argue that as we're all influenced by unintended nudges on an ongoing basis, almost everything we say can act as a nudge of some kind. Therefore, manipulating the nudges to get the best overall health outcome is not only desirable but necessary.

As dentists, we're already in the business of trying to change peoples' behaviour.

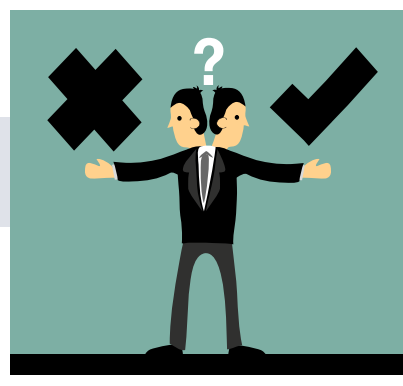

Usually, this takes the form of trying to improve patients' oral hygiene or reduce their sugar intake, but it could go further than this. One can see how easy it would be to coerce patients into accepting more profitable courses of treatment.

There's a risk of patients being exploited for personal gain, but that doesn't mean that we should stop trying to influence behaviour altogether.

We are unable to stop influencing the people we interact with in some way. Therefore we must pay close attention to the wording and phrasing of each of the interactions we have with patients and anything they're likely to see. It is clear that we have a duty to improve the health of our patients, and behaviour modification is one of the best ways we can do that. To do this most effectively, we need to ensure that what we say and do is as effective as possible.

\section{New alumni for dental leadership fellows}

The Health Education England (HEE) (Midlands and East) dental leadership fellows 2018-2019 (pictured) have founded a Dental Leadership Fellow Alumni (DLA) to provide a supportive networking platform for past, present and future clinical leadership fellows.

The HEE clinical and leadership fellowship posts have provided a great opportunity for dentists to become directly involved with leadership and management within the early stages of their careers.

The Alumni aims to promote and support leadership awareness and skills within the dental profession, at both undergraduate and postgraduate levels, through a supportive and sustainable community. It will allow leadership fellows to continue their professional leadership and management development and enable

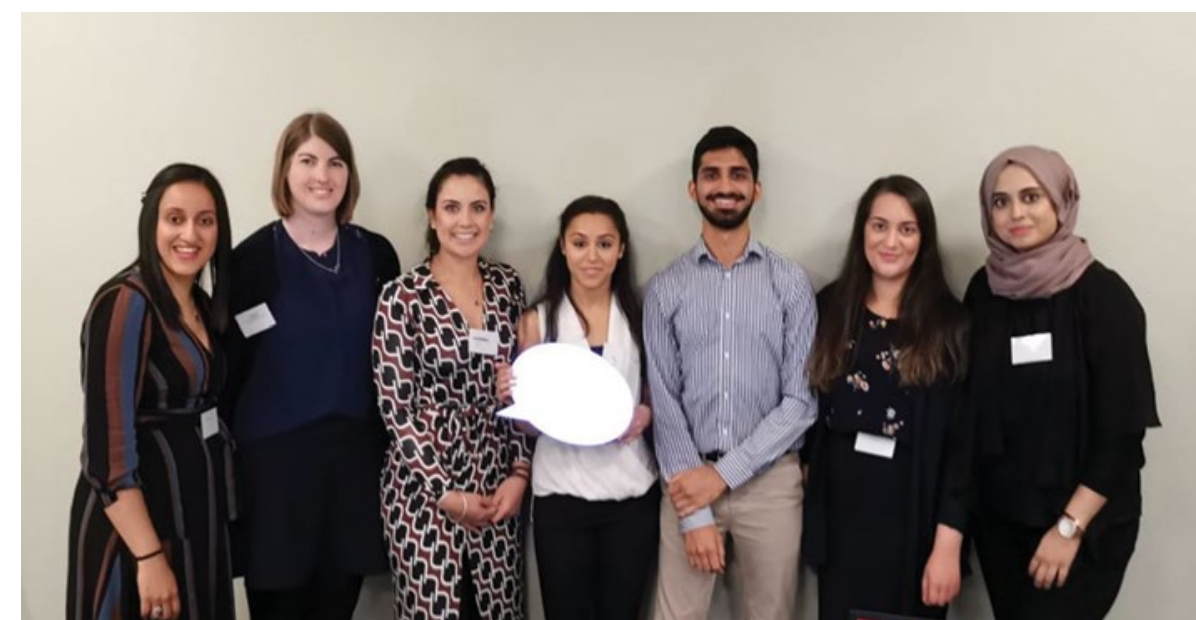

them to remain engaged with the wider leadership community, throughout their career pathways.

To be eligible to join the alumnus scheme, clinicians must have completed the Health
Education England Leadership Fellow scheme and be in good standing with the General Dental/Medical Council.

For more information about the HEE DLA, email leadershipalumni.me@hee.nhs.uk. 